novos produtos

\section{Spectro-Analytical Instruments}

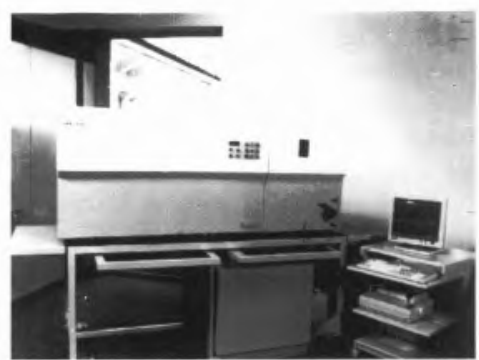

Espectrómetros de emissão óptica por fonte de plasma (ICP), para análise simultânea ou sequencial de elementos em solução.

Novo Spectroflame D, espectrómetro sequencial, com 2 monocromadores, especialmente indicado para laboratórios que analisam muitos elementos em grande número de matrizes.

Contacto:
EN - Equipamento de Análise e Ensaio,
Lda.
Porto - Tels. (02) 322850 - $2026681 / 2$
Fax: (02) 322713
Lisboa - Tels. (01) 3560454 - 3528541
Fax: (01) 3563275

\section{Reómetro Programável modelo DV-III BrookField Software Rheocalc}

Ideal para aplicações de desenvolvimento, pesquisa e controlo de qualidade.

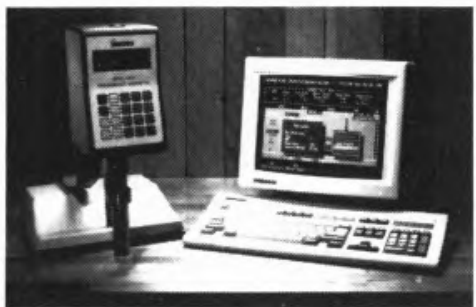

\section{Densímetro Digital Modelo DMA-38 Anton Paar}

Com termostato incorporado, sistema de enchimento e esvaziamento automático.
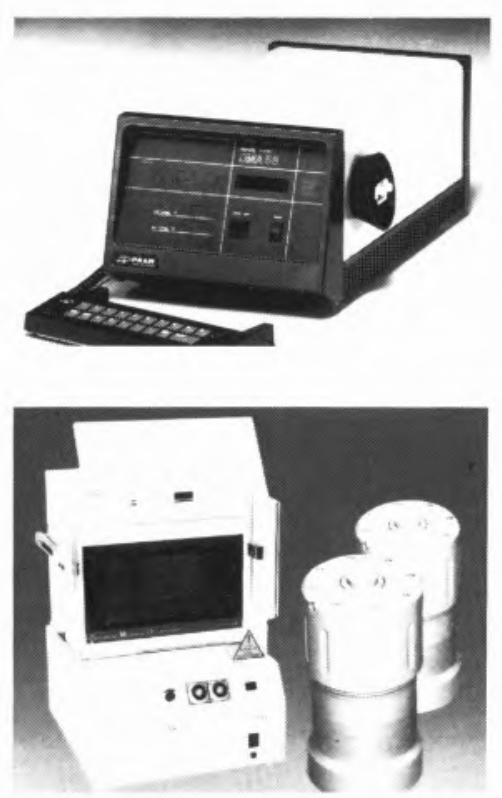

\section{Forno de Micro-Ondas PMD} Anton Paar

Preparação de amostras: Rápida, Segura, Eficaz.
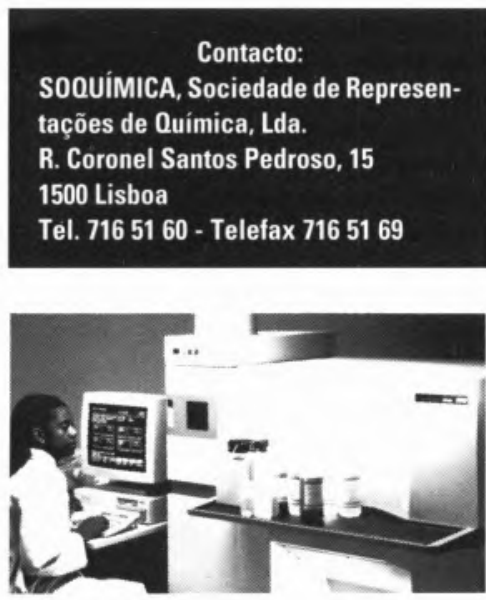

\section{Espectrómetro de Emissão Óptica de Plasma}

0 novo Espectómetro de Emissão Óptica de Plasma indutivo da Perkin Elmer, OPTIMA 3000, representa um salto tecnológico com um novo detector e um novo sistema óptico patenteado que permite medir o background espectral e a linha analítica em simultâneo. Este facto permite determinar 60 elementos em menos de um minuto, a múltiplos comprimentos de onda sem sacrificar a precisão e a sensibilidade. 0 0ptima 3000 inclui 5000 linhas de emissão 0 que permite a selecção de comprimentos de onda livres de interferências espectrais.
As capacidades do Optima 3000 são baseadas no novo detector - desenhado especialmente para emissão óptica -, num sistema óptico e num controle mais eficaz do gerador de RF-free running. 0 espectómetro consiste num policromador tipo Echelle com um detector de SCD - Segmented-array Charged-coupled Detector, um gerador RF free-running $40 \mathrm{MHz}$ com controle verdadeiro de potência (TPC) e pneumática termostatizada-controlada. 0 0ptima 3000 tem uma estrutura compacta, de fácil manutenção e com duas portas para acesso à zona de amostragem.

0 equipamento é totalmente controlado por um computador $\mathbf{4 8 6}$ que automatiza todas as funções incluindo plasma, amostrador automático e aquisição de dados.

0 software permite arquivar as condições de operação mais relevantes, métodos analíticos e calibração.

Durante o desenvolvimento de métodos, a função de optimização relaciona a potência do gerador própria, altura de leitura e fluxos de gases.

0 software permite fazer processamento de resultados pós análise.
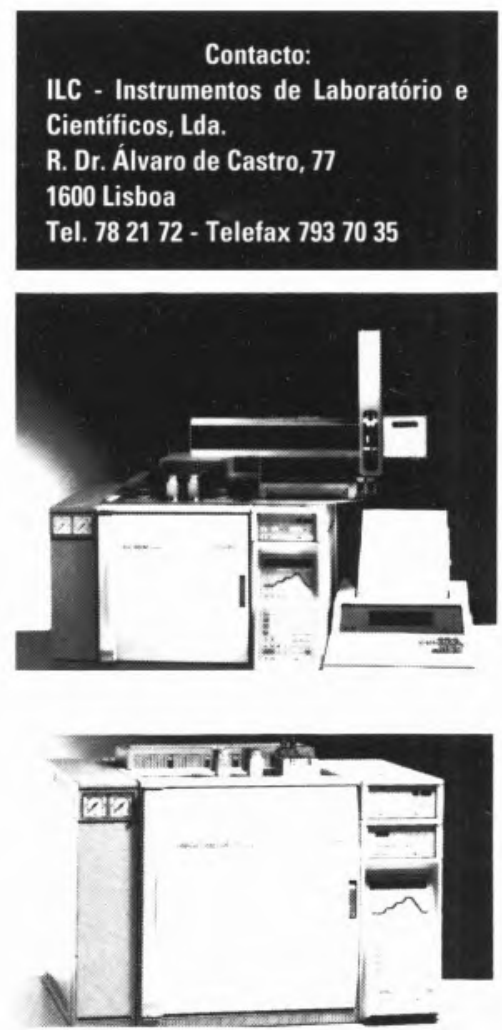

Nova linha de cromatógrafos em fase gasosa da Fisons Instruments SpA

A Fisons Instruments $\mathrm{SpA}$. (previamente Carlo Erba Instruments) acaba de lançar no mercado uma nova série de cromatógrafos em fase gasosa, constituída por 3 
modelos de base, cada um com variadissimas opções.

A gama GC9000 é a mais económica e dedicada a aplicações específicas, tendo no entanto diversas possibilidades de expansão.

A gama GC8000 constitui uma escoIha fácil para quem desejar começar com uma certa configuração e modificá-la ao longo dos anos.

A gama de topo, HRGC MEGA-2 simplesmente o máximo existente actualmente em termos de excelência analítica em cromatografia gasosa capilar.
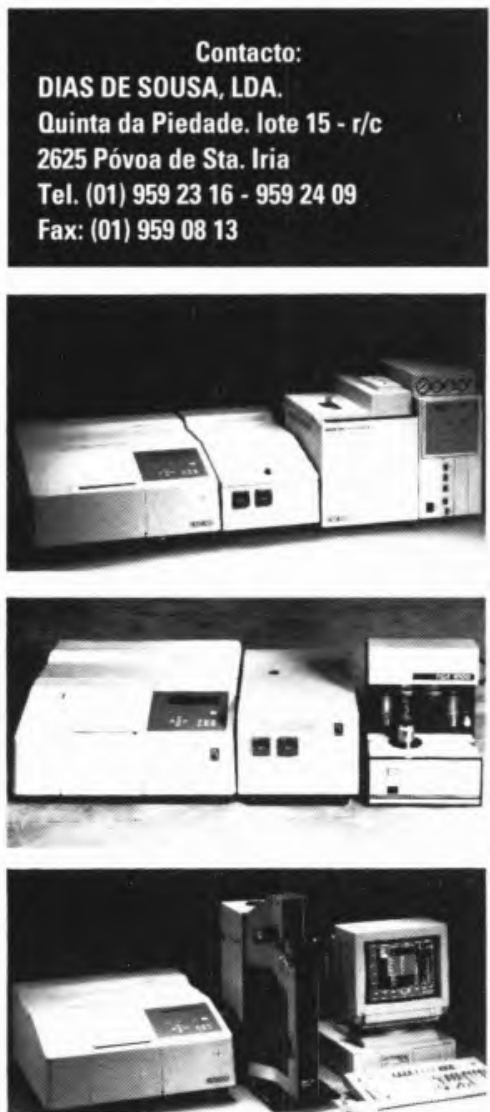

\section{UNICAM - Sistemas Analíticos}

GC - FTIR UNICAM-MATTSON

- Display 3 D, biblioteca e avaliação de espectros

- Display "Real-time" até oito grupos

funcionais

- Velocidades de varrimento variáveis

\section{- TGA / FTIR}

- Linha de transferência de $10 \mathrm{~cm}$ aquecida até $300^{\circ}$

- Software completo de análise para infravermelhos

- MICROSCOPIA FTIR

- Microscópio "QUANTUM" com óptica do

tipơ não cassegrainiana

-Amostragem "real view"

- Detectores MCT ou DTGS

- Análise em transmissão ou reflectância

- Excelente relação preço/qualidade
Contacto:

UNICAM, Sistemas Analiticos

R. Actriz Adelina Fernandes, 13-A

2795 Linda-a-Velha

Tel. 4140325 - Telefax: 4140357

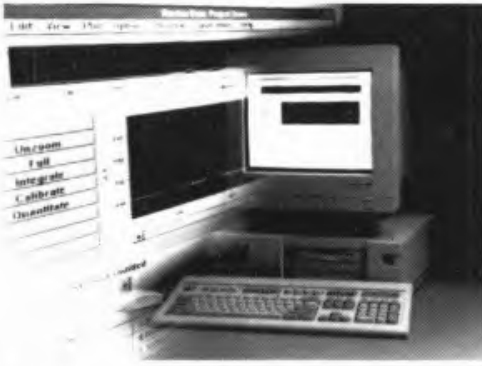

\section{Millenium 2010}

\section{Chromatography Manager.}

Uma nova tecnologia

\section{na gestão de processos}

\section{cromatográficos}

Durante o mês de Março foi apresentado no Porto e em Lisboa uma nova linha de produtos da Waters sob o nome genérico de Millenium.

0 Millenium 2010 Chromatography Manager controla e gere todo o processo cromatográfico, desde a entrada e identificação da amostra até ao relatório final, tudo num só computador. Esta nova tecnologia oferece uma total adaptação às necessidades particulares do laboratório, tanto em departamentos de investigação, como em desenvolvimento de métodos de controlo de qualidade.

Com detectores cada vez mais rápidos e com maior sensibilidade, as análises cromatográficasfornecem uma grande quantidade de dados que tornam problemática a gestão da informação. 0 Millenium 2010 dá resposta a esta necessidade de gestão de dados no laboratório, oferecendo um grau de controlo sem precedentes, sobre toda a informação, melhorando a produtividade do sistema.

Com uma utilização extremamente simples, as diferentes opçōes são mostradas através de ícones, tornando a selecção das operações num processo totalmente intui-

Dispõe de diversas funções exclusivas que simplificam notavelmente o trabalho, destacando-se entre elas:

- oferece a possibilidade de geri todo o sistema cromatográfico, nomeadamente bombas, injectores automáticos detectores;

- a base de dados do Millenium que permite ao utilizador a procura de dados cromatográficos utilizando qualquer tipo de critério:

- gerador de relatórios, que cobre qualquer necessidade na publicação de resultados;

a função de reprocessamento que permite um fácil reprocesso num único écran com um algoritmo exclusivo "Peak Point" que calcula de uma forma automática os parâmetros adequados para a melhor reprodutibilidade e sensibilidade na detecção de picos:

- os procedimentos de trabalho, que permitem transformar os métodos de análise em procedimentos normalizados, eliminando os possiveis desvios introduzidos pelo operador;

- a operação System Suitability/ Confirmação de Métodos, serve para validar tanto 0 sistema como 0 método de análise produzindo um relatório completo com os requisitos estabelecidos nas normas GMP/GLP.

0 Millenium 2010 Chromatography Manager está projectado para cobrir todas as necessidades em HPLC, GPC, GC, FPLC, IC, Electroforese Capilar e Cromatografia Preparativa, em qualquer tipo de laboratório.
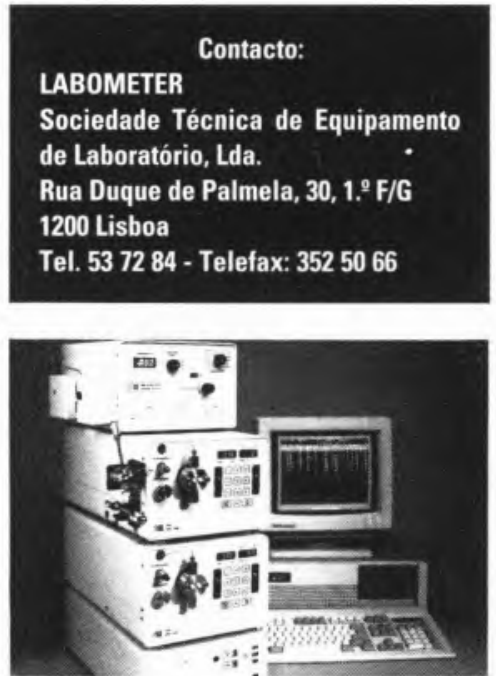

\section{Nova Gama de Cromatógrafos de Líquidos SSI}

Na sequência do processo de consolidação da sua posição de especialistas e líderes de mercado em Cromatografia, KONIK apresenta uma nova gama de cromatógrafos de líquidos de baixo custo, fabricados pela sua Representada Exclusiva SSI (Pennsylvania, EUA).

\section{- Sistemas de HPLC SSI}

SSI oferece uma ampla gama de sistemas modulares para HPLC (sistemas isocráticos, de gradientes ternários a baixa pressão e de gradientes binários a alta pressão), a um baixo custo e suficientemente versáteis para dedicação a cada aplicação nas áreas industrial, laboratorial, investigação e ensino.

\section{- Componentes}

A partir de cada componente SSI ou KONIK (bombas, detectores, controladores de gradientes e sistemas de tratamento de dados), poderão ser criadas uma infinidade de configurações, adequadas a toda e qualquer aplicação em cromatografia de líquidos. Ademais, SSI e KONIK garantem a total compatibilidade dos seus componentes com qualquer sistema de HPLC (Waters e Perkin-Elmer, entre outros), sejam eles injec- 
tores (manual, de injecção repetitiva ou automática), bombas (em aço inox e PEEK), detectores (UV, UV-Vis, Fluorescência, Índice de Refracção, Radioactividade, etc), controladores de gradientes (por teclado ou PC), fornos de colunas (para 3 a 6 colunas) e sistemas de tratamento de dados (registadores, integradores e sistemas baseados em PC).

-Acessórios

SSI fabrica uma completa gama de acessórios (filtros de linha, "check-valves", desgasificadores, conectores, tubos, etc.), em aço inoxe PEEK, compativel com qualquer sistema cromatográfico. Cada acessório para a sua aplicação.

Contacte KONIK INSTRUMENTS para uma demonstração no nosso Laboratório de Aplicações (ou no vosso próprio Laboratório) e tome contacto com a excelência do nosso suporte científico e prestaçōes do nosso equipamento.

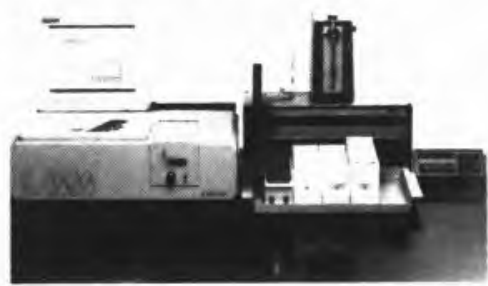

\section{Nova Gama de Espectro- fotómetro SECOMAN}

KONIKINSTRUMENTS tem o prazer de anunciar o recente lançamento da nova linha de espectrofotómetros de SECOMAM (Sarcelles, França).

SECOMAM fabrica desde há mais de 40 anos espectrofotómetros para as mais variadas aplicações, conseguindo conjugar nos seus instrumentos a mais alta performance ao mais baixo custo:

-SECOMAM S.250l, portátil $(7 \mathrm{~kg}) \mathrm{e}$ dispondo de alimentação a 1,110 e $220 \mathrm{~V}$, gama de comprimento de onda de 334 a 900 $\mathrm{nm}$, acessório opcional para amostragem por bomba peristáltica. e mala de transporte resistente ao choque;

-SECOMAM S.500I ( 330 a $900 \mathrm{~nm}) \mathrm{e}$ S.7501 (200 a $1100 \mathrm{~nm})$, dispondo de vários modos de leitura (varrimento, calibração linear e não-linear, cinética com ou sem padrão, absorvância, transmitância e turbidimetria), possibilidade de memorização de métodos (de 24 a 36 métodos, segundo modelo) e acoplamento de vários acessórios, bem como interfaces em série (RS-232C) e em paralelo (impressora), em unidades compactas que permitam o seu fácil transporte $\mathrm{e}$ instalação em qualquer laboratório;

SECOMAM S.1000G2 e S.1000PC unidades de elevada tecnologia, com gama de comprimento de onda de 200 a $1100 \mathrm{~nm}$, dispondo de microprocessador incorporado e permitindo a programação e visualização de 7 métodos espectrofotométricos e controle de acessórios externos, por meio de teclado e monitor próprio (S.1000G2) ou de PC (S.1000PC), apresentando as mais elevadas prestaçōes ao mais baixo custo.

A política de desenvolvimento de produtos seguida por SECOMAM garante a mais elevada qualidade de resultados, aliada à simplicidade de operação, robustez, baixo custo e capacidade de modernização permanente, identificando-se totalmente com a filosofia do Grupo KONIK INSTRUMENTS de apenas desenvolver, fornecer e suportar "EQUIPAMENTOS CAPAZES PARA UTILIZADORES EXIGENTES".

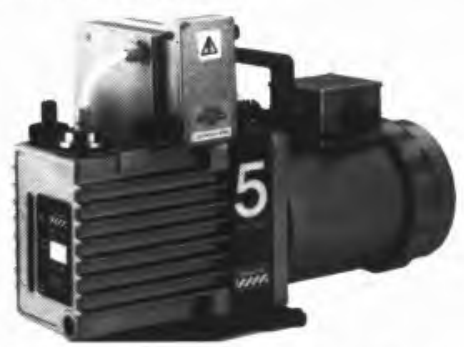

\section{Novas Bombas de Vácuo EDWARDS BUBBLER PUMPS}

EDWARD HIGH VACUUM (CRAWLEY, Inglaterra), representada em exclusividade na Península Ibérica pelo Grupo KONIK INSTRUMENTS, lançou recentemente uma nova geração de três novas bombas, denominadas "Bubbler Pumps", para aplicações onde excessivas quantidades de solventese vapor de água são produzidos.

Como se sabe, as bombas rotativas são ideais, pela sua performance, para aplicações tais como a secagem de gel, electroforese, destilação, liofilização, concentração de solventes e centrifugação.

No entanto, tais processos geram elevadas quantidades de vapores (solventes e/ou água, frequentemente excedendo a capacidade de evacuação da bomba, e em particular se a bombagem é iniciada antes de atingida a temperatura óptima de utilização. 0 vapor em excesso tende a condensar no interior da bomba de vácuo, degradando o óleo lubrificante e expondo o interior da bomba ao ataque do condensado, o qual pode ser corrosivo.

As novas EDWARDS Bubbler Pumps incorporam um acessório especial (borbuIhador), o qual mantém o óleo lubrificante permanentemente condicionado, aumentando a capacidade da bomba de vácuo em remover vapores, mantendo a sua elevada performance. Mesmo quando a bomba é utilizada intermitantemente, poderá com toda a segurança deixar-se em "stand-by", enquanto o "borbulhador" (bubbler) mantém-se em funcionamento, removendo os vapores e evitando o risco de desligar-se a bomba de vácuo deixando-a vulnerávelà contaminação do seu óleo lubrificante e corrosão das suas partes internas.

\begin{tabular}{l}
\multicolumn{1}{c}{ Contacto: } \\
KONIK INSTRUMENTS, SA \\
Rua do Conde Redondo, 13-A - c/v \\
1100 Lisboa \\
Tel. (01) 575787 - Fax: (01) 575847 \\
ou Porto \\
Tel. (02) 6068855 - Fax: (02) 6002135
\end{tabular}

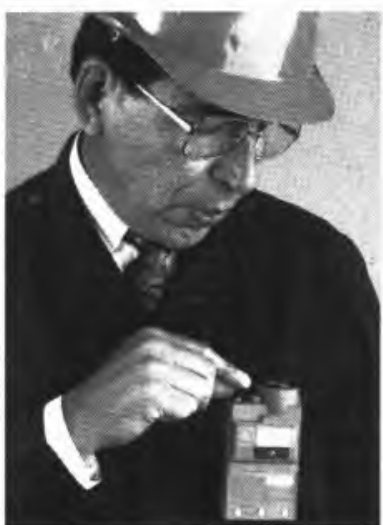

Nova Gama de monitores pessoais de gás

A CROWCON DETECTION INSTRUMENTS LIMITED acaba de lançar uma nova gama de monitores pessoais de gás pequenos e leves. A gama GASMAN comporta modelos para detecção dos gases tóxicos comuns, oxigénio e uma versão para gases inflamáveis. Medindo apenas $115 \times 66 \times 39$ mme pesando no máximo $268 \mathrm{~g}$, os monitores GASMAN são concebidos para serem transportados no bolso da camisa ou presos ao cinto pelo clip incorporado. Para assegurar a detecção imediata de um gás perigoso, os sensores são colocados no topo do instrumento. Quando existe uma situação perigosa, soa um alarme pulsante de $77 \mathrm{~dB}$ e pisca um LED vermelho. As concentrações de gás são lidas num mostrador digital com iluminação posterior. Os modelos GASMAN TOX têm alarmes ajustados para actuar no momento em que o limite de exposição ocupacional (OEL)de 8 horas é atingido. Quando requerido, podem ser fornecidos com alarmes para média pesada em tempo (TWA). 0 GASMAN $\mathrm{OX}$ tem dois niveis de alarme para prevenir contra deficiência ou excesso de oxigénio, enquanto que o GASMAN FL alerta o utilizador quando a concentração de gás inflamável atinge 20\% LEL (limite explosivo inferior).

Outras características incluem a indicação por LED de que o monitor está a funcionar correctamente e um aviso de bateria fraca. A alimentação é fornecida por quatro pilhas tipo AA que terão uma duraçāo aproximada de quatro meses com uma operação normal de 40 horas semanais, existindo, opcionalmente, conjuntos de baterias recarregáveis. Todos os monitores da gama GASMAN são intrinsecamente seguros. Desenhadas para suportar os rigores do uso industrial, as caixas são resistentes à água e à poeira, de acordo com IP65. Para ambientes extremamente poeirentos poderá ser fornecida uma caixa de transporte opcional.

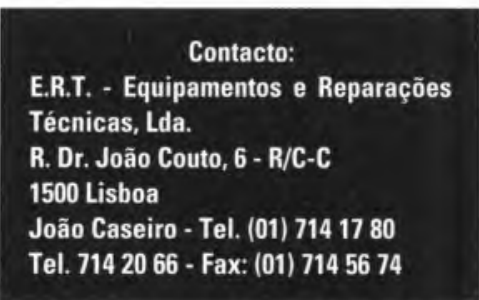

